\title{
Double Solitaire Mechanical Thrombectomy in Acute Stroke: Effective Rescue Strategy for Refractory Artery Occlusions?
}

\author{
J. Klisch, V. Sychra, C. Strasilla, C.A. Taschner, M. Reinhard, H. Urbach, and DS. Meckel
}

\begin{abstract}
BACKGROUND AND PURPOSE: Mechanical thrombectomy by using a single stent retriever system has demonstrated high efficacy for recanalization of large-artery occlusions in acute stroke. We aimed to evaluate the feasibility, safety, and efficacy of a novel double Solitaire stent retriever technique as an escalating treatment for occlusions that are refractory to first-line single stent retriever mechanical thrombectomy.
\end{abstract}

MATERIALS AND METHODS: All patients treated with the double stent retriever technique by using the Solitaire system were retrospectively selected from 2 large neurointerventional centers. Time to recanalization, angiographic (TICI) and clinical outcomes (mRS), and complications were assessed.

RESULTS: Ten patients (median NIHSS score, 16; mean age, 70 years) with MCA M1 segment $(n=5)$ and terminal ICA ( $n=5$ including 2 ICA tandem) occlusions were included. Prior single stent retriever mechanical thrombectomy had been performed in 9 patients (median number of passes, 3). Median time to recanalization was 60 minutes (interquartile range, $45-87$ minutes). Procedure-related complications occurred in 1 patient; overall mortality was $20 \%$. Recanalization of the target vessel $(\mathrm{TICl} 2 \mathrm{~b} / 3$ ) was achieved in $80 \%$. Good clinical outcome (mRS 0-2) was $50 \%$.

CONCLUSIONS: In this preliminary feasibility study, the double Solitaire stent retriever technique proved to be an effective method for recanalization of anterior circulation large-artery occlusions refractory to standard stent retriever mechanical thrombectomy.

ABBREVIATIONS: DAC = distal-access catheter; ICAT $=$ internal carotid artery terminus; $M T=$ mechanical thrombectomy; $\mathrm{SR}=$ stent retriever

I acute ischemic stroke, recanalization of an occluded cerebral artery is strongly linked with improved clinical outcome and reduced mortality. ${ }^{1}$ The potential of IV thrombolysis for achieving successful vessel recanalization is significantly limited by the extent of clot burden in proximal cerebral artery occlusions. ${ }^{2}$ With the recent introduction of stent retrievers (SR) for mechanical thrombectomy (MT), fast, safe, and efficient large-artery recanalization treatment can be achieved, and their superiority over older MT devices has been demonstrated in randomized controlled trials. ${ }^{3,4}$

Despite considerable recanalization rates of $61 \%-86 \%$ (Thrombolysis in Myocardial Infarction/TICI scores of $\geq 2 / 2 b$ ), ${ }^{3-5}$ up to

Received June 29, 2014; accepted after revision August 13.

From the Institute of Diagnostic und Interventional Radiology and Neuroradiology (J.K., V.S., C.S.), Helios Klinikum, Erfurt, Germany; and Departments of Neuroradiology (C.A.T., H.U., S.M.) and Neurology (M.R.), University Hospital Freiburg, Freiburg, Germany.

Please address correspondence to Stephan Meckel, MD, Department of Neuroradiology, University Hospital Freiburg, Breisacher Str 64, D-79106 Freiburg, Germany; e-mail: stephanmeckel@gmail.com

三 Indicates article with supplemental on-line table.

http://dx.doi.org/10.3174/ajnr.A4133
$33.3 \%$ of patients are still left without sufficient recanalization after standard SR MT. ${ }^{6}$ In these refractory cases, different rescue treatments have been proposed with variable rates of success. $^{4,5,7-10}$ These include local intra-arterial fibrinolysis, MT with the Penumbra device (Penumbra, Alameda, California), mechanical thrombus disruption, thromboaspiration through a distal-access catheter (DAC), balloon angioplasty, and/or stent placement.

Here, we describe a novel escalating strategy for MT by using 2 Solitaire SR devices (Covidien, Irvine, California), hence termed the "double Solitaire SR technique," for proximal anterior circulation occlusions that are refractory to first-line single SR MT. In a retrospective series of patients from 2 large neurointerventional centers, we assessed the feasibility, safety, and angiographic and clinical outcomes of this technique.

\section{MATERIALS AND METHODS \\ Patient Selection}

In this retrospective study, a consecutive series of patients was identified from in-hospital stroke databases that included patients with acute stroke due to large cerebral artery occlusions undergo- 
ing MT treatments. We collected all patients treated with the double Solitaire SR technique between its introduction in December 2012 and May 2014 from 2 large tertiary neurointerventional centers. At both centers, a total of 234 MT treatments for anterior circulation occlusions were performed. The eligibility criteria for these MT treatments were the following: 1) NIHSS $\geq 8$; 2) confirmed proximal anterior circulation cerebral artery occlusion (ICA including tandem lesions, eg, proximal ICA stenosis and ICA terminus [ICAT] or the MCA M1 segment) with a TICI score of 0 or 1; and 3) initiation of MT treatment within 6 hours of symptom onset. In general, written consent is not obtained from all patients undergoing MT treatments in the emergency setting of an acute stroke at both involved centers. The choice of MT treatment is made as an interdisciplinary decision between the on-call attending neurointerventionalist and neurologist on an individual basis, considering relevant inclusion/exclusion criteria that are documented in the patient's file. Approval by the ethics committee was waived because the double Solitaire SR technique represented a rescue treatment in case of failure of the approved standard MT technique using a single SR device, which was performed at the individual operator's decision (see below).

Baseline investigations included the assessment of NIHSS by an experienced on-call stroke neurologist, independent of this study. When no other contraindications were present, a bridging IV thrombolysis treatment was started directly after cranial imaging.

\section{MT Procedures}

All procedures were performed with the patient under general anesthesia. Standard first-line MT with a single SR was performed either by using a balloon-guide catheter or a DAC as described previously. ${ }^{11-13}$ The Solitaire device was used in all MT procedures either as a single device or for the double SR technique. The sizes of the Solitaire devices used included Solitaire AB, $3 \times 20$ $\mathrm{mm}$, and Solitaire FR or Solitaire Two, $4 \times 20 \mathrm{~mm}$ and $6 \times 30$ $\mathrm{mm}$. In case of insufficient target-vessel recanalization (TICI score, 0 and 1), the SR sequence was repeated on the basis of the individual operator's decision until the double Solitaire SR technique was initiated. This decision was influenced by several factors, including clot load/length of the occluded arterial segment, size and composition of the already retrieved clot fragments, the amount of clot extension into the bifurcation limbs, and the duration of multiple single SR passages.

\section{Double Solitaire SR Technique}

With the use of an $8 \mathrm{~F}$ balloon-guide catheter (Merci; Concentric Medical, Mountain View, California; or Cello; Covidien), two 0.021-inch internal-diameter microcatheters (Prowler Select Plus; Codman \& Shurtleff, Raynham, Massachusetts; or Rebar18; Covidien) were navigated sequentially into the occluded vessel. They were either placed parallel to each other with at least 1 tip distal to the thrombus or in a Y-type configuration with both tips beyond the thrombus, ending in separate bifurcation limbs (eg, the superior and inferior MCA trunks). The parallel configuration of the SRs may enable retrieval of large and/or long thrombi, with both SR devices placed beside each other, also allowing distal device overlap. The latter may potentially increase the area of thrombus catchment in long segmental occlusions extending from the MCA bifurcation to the terminal ICA. Then, 2 Solitaire devices of similar or different sizes (eg, $4 \times 20$ and $6 \times 30 \mathrm{~mm}$ ) were delivered from each microcatheter in a sequential fashion to cover the whole extent of the thrombus. A control angiography was performed after unfolding the SRs to evaluate re-establishment of flow. Both SRs were left deployed for 3-10 minutes depending on the degree of flow in the target vessel at the individual operator's discretion. The detachment zones of both SR devices were covered by the tips of the microcatheters to prevent inadvertent detachment during the retrieval maneuver. Then, both devices were slowly recovered by simultaneously pulling them together into the balloon-guide catheter under continuous aspiration and fluoroscopic imaging control. After recovery, both stent devices were found entangled in each other, in some cases with the thrombus embedded between the struts; however, they usually could be carefully released and cleaned of thrombus fragments.

With the use of a DAC (Penumbra 54; Penumbra; or Navien 0.058; Covidien), parallel placement of 2 microcatheters is not possible. Therefore, 1 microcatheter was first placed with its tip distal enough within 1 arterial limb so that the proximal end of the SR did not cover the bifurcation, to avoid an inadvertent placement of the second SR through the struts of the first one. After unfolding the first SR, we removed the microcatheter completely from the DAC, leaving a bare Solitaire device inside the DAC. Then, the second bifurcating limb was catheterized with a microcatheter, and the second SR device was unfolded in a standard fashion. The detachment zone of the proximal SR was left covered by the tip of the microcatheter so that in case of inadvertent detachment of the more distally placed SR during retrieval, the proximal one could still be recovered. Both SRs were subsequently recovered as described above while aspiration was performed directly through the DAC. Thereby, the tip of the DAC was placed as close as possible to the clot (eg, into the terminal ICA or orifice of the M1 segment, depending on the length of the clot), to achieve an optimal aspiration result without clot fragmentation during the retrieval maneuver.

The time from symptom onset to the start of the MT procedure (first angiographic image) and total recanalization time (first angiographic image to the image demonstrating final recanalization) were recorded.

\section{Outcome Analysis and Follow-Up}

The degree of target-vessel recanalization was assessed by using the TICI score by 2 experienced interventional neuroradiologists independently from the procedures. Functional outcome was assessed by using the mRS score on discharge from the rehabilitation center (usually at days 60-90 after stroke onset) from respective patient files, determined by experienced neurologists blinded to the treatment details of the double Solitaire SR procedure. Furthermore, we recorded the following procedural parameters: use of a DAC or balloon-guide catheter, use of a bridging IVT, use of carotid stent placement for treatment of proximal ICA stenosis/ occlusion, the number of unsuccessful single SR passes before application of the double SR technique, additional escalating techniques, and complications associated with MT procedures including proce- 


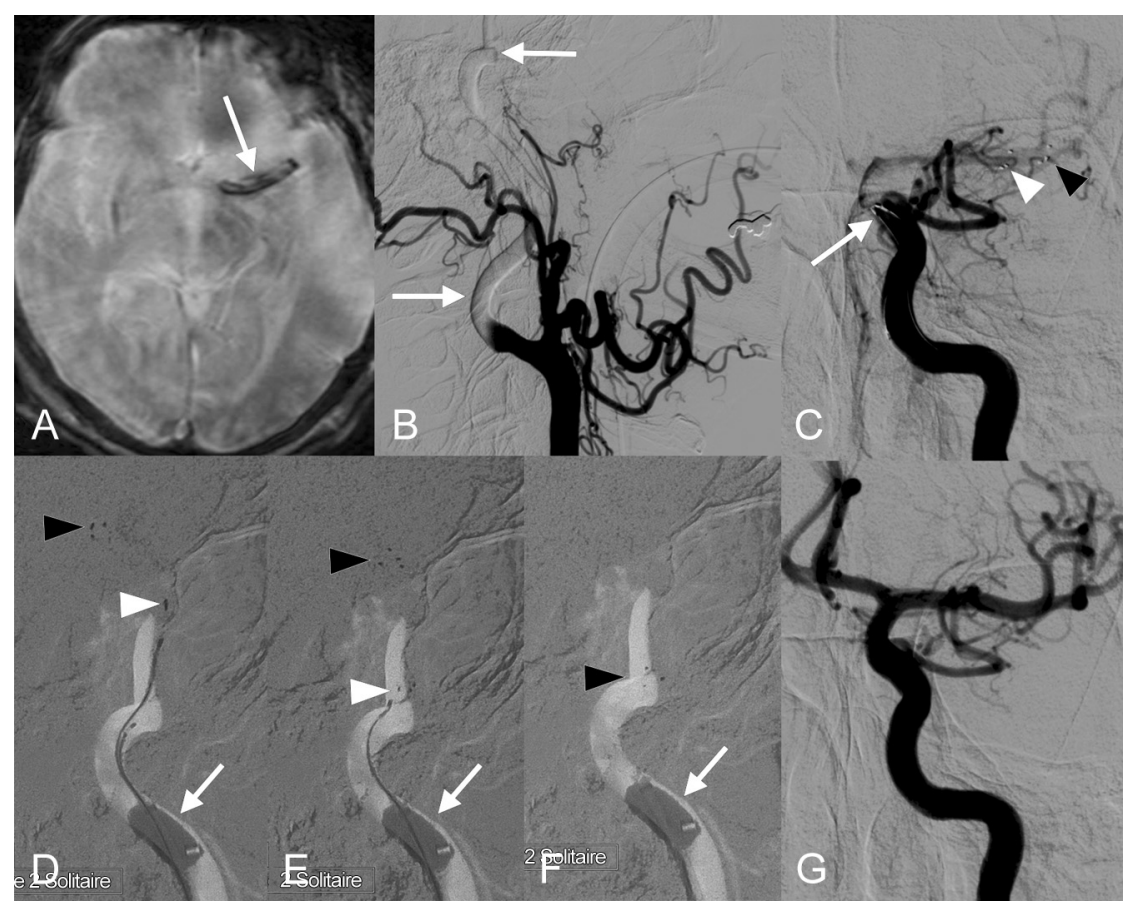

FIG 1. Double Solitaire SR maneuver in patient 1 with a left ICAT occlusion. Large M1 thrombus (arrow in A, gradient recalled-echo image) and proximal ICA contrast media stasis (arrows in B) are shown. Two Solitaire SRs are placed parallel (arrow in C, proximal markers) with 1 extending to the MCA bifurcation (black arrowhead, $6 \times 30 \mathrm{~mm}$ ) and 1 into the mid-M1 segment (white arrowhead, $4 \times 20 \mathrm{~mm}$ ). Three consecutive fluoroscopic images $(D-F)$ depict simultaneous retrieval of both SRs; during this maneuver, the distal tip markers of the longer SR device (black arrowheads) follow the shorter one (white arrowheads), with retrieval of a large thrombus inside the tip of the inflated balloon-guide catheter (arrows). After double Solitaire SR MT, complete recanalization is demonstrated $(G)$.

dural complications and postprocedural hemorrhage of any cause associated with a poor clinical outcome ( $\mathrm{mRS}>2)$.

\section{RESULTS}

We included 10 consecutive patients ( 7 men, 3 women; mean age, 70 years; range, $51-88$ years). These included 5 patients treated for MCA M1 occlusion; 3, for ICAT occlusion; and 2, for ICA tandem occlusion, which overall represented $4.3 \%$ of all patients undergoing anterior circulation MT procedures during the analyzed time (see above). The median baseline NIHSS score was 16 (interquartile range, 13-19), and the median time from symptom onset to the start of the MT procedure was 168 minutes (interquartile range, 150-179 minutes). For both ICA tandem occlusions, proximal ICA stent placement was performed before MT. Nine patients were treated by a single SR MT before the double Solitaire SR technique, with a median number of 3 (range, 1-4) unsuccessful passes. In 1 patient (patient 9), the double Solitaire SR technique was performed as a primary MT treatment to avoid thrombus fragmentation during retrieval because superselective microcatheter injection had demonstrated exceptionally long thrombi extending from the ICAT via M1 to both the superior and inferior M2 trunks. The details of all patients including therapy, complications, and outcome are summarized in the On-line Table.

With the double Solitaire SR technique, both SRs were placed in a parallel configuration from M1 into the terminal ICA in 1 patient (Fig 1). In the remaining 9 patients, a Y-type SR configuration was used (Fig 2). Of these, both SRs extended into $2 \mathrm{MCA}$ divisions in 8 patients and into the M1 and $\mathrm{A} 1$ segments in 1 patient (patient 8 ). Before retrieval of both SRs, control angiograms disclosed variable degrees of reestablished target-vessel flow in all patients. In 8 patients, a single pass with both SRs was performed. In 2 patients (patients 7 and 9), the double Solitaire SR technique was applied twice. In 2 patients, we performed additional escalating therapies: In patient 4 , a residual M2 trunk occlusion was treated with an intravenous bolus of eptifibatide (glycoprotein IIb/ IIIa antagonist) and another pass with a single SR. In patient 8 , a refractory ICAT occlusion due to an underlying terminal ICA stenosis was finally treated with unsuccessful balloon angioplasty (a noncompliant balloon was used because wall-adherent thrombus and partially thrombosed atherosclerotic stenosis could initially not be discerned), and stent placement could not be performed due to difficult vascular access.

The median total recanalization time was 60 minutes (interquartile range, 45-87 minutes), including all additional procedures such as ICA stent placement. Overall complete recanalization (TICI $2 \mathrm{~b} / 3$ ) was achieved in 8 patients (80\%), and good clinical outcome $(\mathrm{mRS} \leq 2)$ was observed in 5 patients (50\%). There were 2 fatalities (20\%): One was a related malignant infarct following failed recanalization of an ICAT occlusion (patient 8 , see above). The other (patient 6) had successful recanalization of an ICAT occlusion with initial neurologic improvement and then secondary deterioration 3 weeks later, with aphasia and hemiparesis due to presumed re-infarction (it was not further investigated due to the patient's documented provision, which only became apparent after the initial treatment).

We observed 1 procedural complication: Patient 4 had a residual proximal M2 occlusion after 2 unsuccessful single SR passes and 1 double SR pass. Contrast extravasation from the M2 segment was observed after further therapy escalation with IV eptifibatide and another single SR pass (see above). The ruptured segment was temporarily occluded with a single coil until active bleeding had stopped angiographically. Following decompressive craniectomy due to a malignant MCA infarct the next day, the outcome was poor (mRS 4). Inadvertent SR detachment during retrieval of both devices was not observed.

Mild vasospasm after the double Solitaire SR maneuver was encountered in 7 patients (70\%). No severe vasospasm or arterial dissection was encountered.

\section{DISCUSSION}

Our experience with this new off-label simultaneous use of 2 Solitaire SRs has been encouraging due to its ease of use and ability to achieve effective recanalization in refractory cases (TICI $2 \mathrm{~b} / 3$ in $60 \%$ and 


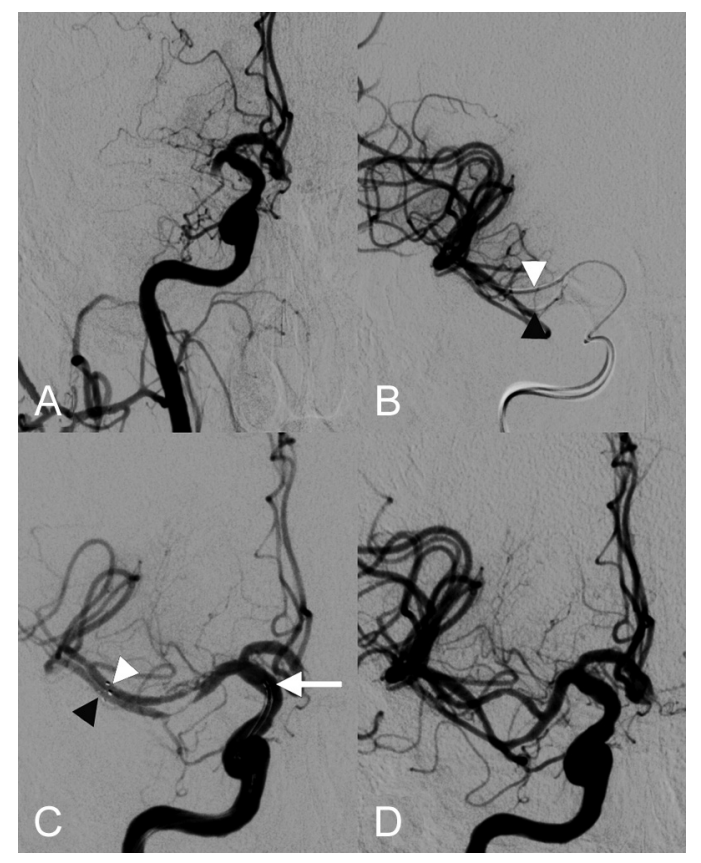

FIG 2. Double Solitaire SR maneuver with SRs in a Y-type configuration in patient 2 with MCA Ml occlusion. Following 3 single SR passes, there is refractory $\mathrm{Ml}$ occlusion $(A)$. Simultaneous injection of 2 microcatheters being placed in the superior (white arrowhead) and inferior (black arrowhead) MCA divisions is shown in B. C, ICA injection after unfolding of 2 Solitaire devices $(4 \times 20 \mathrm{~mm})$, which extend from both $M 2$ segments (distal markers in the superior and inferior divisions, white and black arrowheads, respectively) to the terminal ICA (attachment zones, white arrow), demonstrates re-establishment of flow within both MCA trunks. $D$, After simultaneous retrieval of both SRs by using proximal occlusion and aspiration through a balloon-guide catheter, both MCA trunks are recanalized. Mild vasospasm of ICA and MCA vessels is depicted.

$80 \%$ after 1 and 2 passes with the double Solitaire SR technique, respectively). The median total recanalization times of 60 minutes (interquartile range, 45-87 minutes) were increased compared with those reported in standard MT procedures with a single SR (range, 20-48 minutes). ${ }^{3,5,9-11}$ However, given that these procedural times also included a median number of 3 unsuccessful single SR passes, the extra procedural time appears tolerable. The overall good clinical outcome (50\%) was comparable with the results of larger series/trials with the standard single SR MT technique (40\%-58\%). ${ }^{3-5}$ The Ytype configuration of the 2 SRs appears particularly useful for retrieving riding emboli at arterial bifurcations such as M1 emboli that extend into both M2 trunks. This technique may also be applied to refractory basilar artery occlusions due to emboli extending into both posterior cerebral arteries. Likewise, Clarençon et al ${ }^{14}$ reported a case of an acute basilar artery occlusion that was treated successfully with double Merci retriever devices in a Y-type configuration via a bilateral vertebral artery approach.

In previous studies, other rescue therapies were usually applied in patients in whom adequate recanalization (TICI $\geq 2 \mathrm{~b}$ ) could not be achieved following several passes with a single SR (usually 3-5 passes at the individual operator's discretion). ${ }^{3-5,9,11}$ However, the success rates of various rescue treatments including intra-arterial pharmacologic thrombolysis, other MT devices, thromboaspiration (Penumbra), balloon angioplasty, or intracranial stent placement remain limited. ${ }^{5,9-11}$ For example, patients who received a rescue therapy in the Solitaire FR Thrombectomy for Acute Revascularization (STAR) study showed a significantly lower rate of favorable outcome compared with those who did not (33.3\% versus $60.3 \%) .{ }^{5}$ Likewise, Kurre et al ${ }^{10}$ recently reported a significantly lower rate of adequate recanalization in patients who had rescue treatments compared with those with a maximum of 3 passes with a single SR device.

Cervical or intracranial vessel trauma remains a potentially serious procedure-related adverse event, which occurs at a low incidence with the use of the standard single SR MT technique. ${ }^{3,5,10,15,16}$ In the Solitaire FR With the Intention For Thrombectomy trial, the reported incidences of arterial dissection, symptomatic cerebral hemorrhage, and SAH were $4.5 \%$, $1.1 \%$, and $1.1 \%$, respectively. ${ }^{15}$ The use of 2 parallel SRs within the MCA or ICA vessel lumen may potentially increase the force on the endothelium during the retrieval maneuver. We observed only 1 vessel trauma occurring after several single SR passes, 1 double SR pass, and further escalating therapy (see above). Within the limited experience of this feasibility study, therapeutic escalation beyond the double Solitaire SR technique should thus be made with caution.

In our series, we included a single case in which the double Solitaire SR technique was performed as a primary treatment for MT of an ICAT occlusion due to exceptionally long thrombi. However, on the basis of these preliminary results, this technique should be reserved only as a rescue treatment for cases refractory to standard single SR MT until further data on the safety and outcome are available. In general, the decision to extend the procedure after several unsuccessful retrieval attempts with a single SR device should only be made after careful consideration of individual factors defining the likelihood of a clinical success. Such factors may include the time from stroke onset, the extent of baseline infarct core, or the presence of underlying intracranial artery stenosis. The application of this technique with a second SR device will potentially add a significant increase in hospital costs to an already expensive acute stroke treatment. On the other hand, this may be considered worthwhile in light of high costs for care in cases of futile recanalization therapy resulting in a poor clinical outcome. However, the analysis of cost effectiveness was far beyond the scope of this feasibility study.

The limitations of the study relate to its small sample size and retrospective nature, which was designed to provide evidence of the feasibility and safety of this novel rescue technique for MT. The angiographic and clinical outcome data are promising but require further confirmation in larger prospective studies to demonstrate a possible improvement in clinical outcome related to the application of this MT escalating strategy compared with standard single SR MT only. Until the risk/benefit ratio has been further determined in such studies, practitioners are advised to proceed with extreme caution when applying this technique.

\section{CONCLUSIONS}

In this preliminary feasibility study, the double Solitaire SR technique for MT therapy proved to be an effective rescue method for rapid recanalization of refractory anterior circulation occlusions. The technique showed a low complication rate and a high rate of target-vessel recanalization with 50\% favorable clinical outcome. Further pro- 
spective studies are required to demonstrate the clinical benefit for the subgroup of patients with refractory cerebral artery occlusion.

Disclosures: Joachim Klisch—UNRELATED: Consultancy: Sequent Medical. ${ }^{*}$ Christian A. Taschner-UNRELATED: Board Membership: MircoVention, ${ }^{*}$ Acandis*; Consultancy: Stryker Neurovascular. Stephan Meckel—UNRELATED: Travel/Accommodations/Meeting Expenses Unrelated to Activities Listed: Stryker, MicroVention, Comments: travel expenses and Congress fees. *Money paid to the institution.

\section{REFERENCES}

1. Rha JH, Saver JL. The impact of recanalization on ischemic stroke outcome: a meta-analysis. Stroke 2007;38:967-73

2. Riedel CH, Zimmermann P, Jensen-Kondering $\mathrm{U}$, et al. The importance of size: successful recanalization by intravenous thrombolysis in acute anterior stroke depends on thrombus length. Stroke 2011;42:1775-77

3. Nogueira RG, Lutsep HL, Gupta R, et al. Trevo versus Merci retrievers for thrombectomy revascularisation of large vessel occlusions in acute ischaemic stroke (TREVO 2): a randomised trial. Lancet 2012;380:1231-40

4. Saver JL, Jahan R, Levy EI, et al. Solitaire flow restoration device versus the Merci retriever in patients with acute ischaemic stroke (SWIFT): a randomised, parallel-group, non-inferiority trial. Lancet 2012;380:1241-49

5. Pereira VM, Gralla J, Davalos A, et al. Prospective, multicenter, single-arm study of mechanical thrombectomy using Solitaire flow restoration in acute ischemic stroke. Stroke 2013;44:2802-07

6. Gascou G, Lobotesis K, Machi P, et al. Stent retrievers in acute ischemic stroke: complications and failures during the perioperative period. AJNR Am J Neuroradiol 2014;35:734-40

7. Brekenfeld C, Schroth G, Mordasini P, et al. Impact of retrievable stents on acute ischemic stroke treatment. AJNR Am J Neuroradiol 2011;32:1269-73
8. Miteff F, Faulder KC, Goh AC, et al. Mechanical thrombectomy with a self-expanding retrievable intracranial stent (Solitaire $\mathrm{AB}$ ): experience in 26 patients with acute cerebral artery occlusion. AJNR Am J Neuroradiol 2011;32:1078-81

9. Yoon YH, Yoon W, Jung MY, et al. Outcome of mechanical thrombectomy with Solitaire stent as first-line intra-arterial treatment in intracranial internal carotid artery occlusion. Neuroradiology 2013;55:999-1005

10. Kurre W, Aguilar-Perez M, Schmid E, et al. Clinical experience with the pREset stent retriever for the treatment of acute ischemic stroke-a review of 271 consecutive cases. Neuroradiology 2014;56: 397-403

11. Dávalos A, Pereira VM, Chapot R, et al. Retrospective multicenter study of Solitaire FR for revascularization in the treatment of acute ischemic stroke. Stroke 2012;43:2699-705

12. Roth C, Papanagiotou P, Behnke S, et al. Stent-assisted mechanical recanalization for treatment of acute intracerebral artery occlusions. Stroke 2010;41:2559-67

13. Möhlenbruch M, Seifert M, Okulla T, et al. Mechanical thrombectomy compared to local-intraarterial thrombolysis in carotid $\mathrm{T}$ and middle cerebral artery occlusions: a single center experience. Clin Neuroradiol 2012;22:141-47

14. Clarençon F, Blanc R, Gallas S, et al. Thrombectomy for acute basilar artery occlusion by using double Merci retriever devices and bilateral temporary vertebral artery flow reversal: technical note. J Neurosurg 2009;111:53-56

15. Akins PT, Amar AP, Pakbaz RS, et al. Complications of endovascular treatment for acute stroke in the SWIFT trial with Solitaire and Merci devices. AJNR Am J Neuroradiol 2014;35:524-28

16. Gory B, Bresson D, Kessler I, et al. Histopathologic evaluation of arterial wall response to 5 neurovascular mechanical thrombectomy devices in a swine model. AJNR Am J Neuroradiol 2013;34: 2192-98 